\title{
Synthesis of Chromen-2-one, Pyrano[3,4-c]chromene and Pyridino[3,4-c]chromene Derivatives as Potent Antimicrobial Agents
}

\author{
Sawsan A. Fouad, ${ }^{1}$ Sadia A. Hessein, ${ }^{1}$ Samir $Y$. Abbas, ${ }^{2}$ Amel M. Farrag, ${ }^{3}$ Yousry A. Ammar ${ }^{4, *}$
}

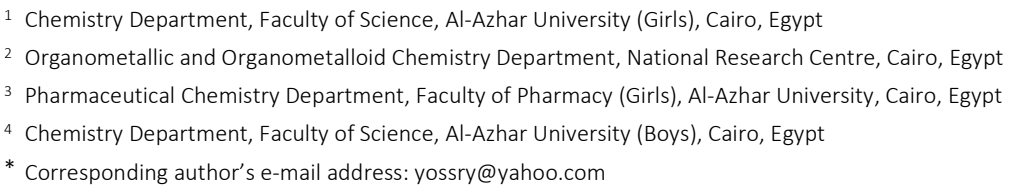

RECEIVED: December 18, 2017 * REVISED: April 18, 2018 * ACCEPTED: April 22, 2018

Abstract: In an attempt for development of new antimicrobial agents; new series of chromen-2-one, pyrano[3,4-c]chromene and pyridino[3,4c]chromene derivatives bearing a diazo moiety were synthesized. Chromen-2-one derivatives were synthesized via treatment of 5-(aryldiazo) salicylaldehyde with different type of active methylene derivatives. Pyrano[3,4-c]chromene and pyridino[3,4-c]chromene derivatives were synthesized via treatment of chromen-2-one derivatives with another active methylene derivatives. The synthesized compounds were evaluated for their expected antimicrobial activity; where, the majority of these compounds showed potent antibacterial and antifungal activities against the tested strains of bacteria and fungi.

Keywords: Chromen-2-ones, Pyrano[3,4-c]chromenes, Pyridino[3,4-c]chromene; Antibacterial and antifungal activities.

\section{INTRODUCTION}

$\mathbf{N}$ ATURAL and synthetic chromen-2-one derivatives have great interest from medicinal chemists, many of the chromene derivatives used as precursors for the synthesis of potentially biologically active compounds. ${ }^{[1-5]}$ Chromen-2-one moiety is being the parent scaffold in many of biological derivatives. Some of chromene skeleton are well established as antimicrobial agents such as Novobiocin and Chlorobiocin. [6] Recently, chromene derivatives have great interesting due to their potential application such as anti-HIV, ${ }^{[7]}$ antitubercular, ${ }^{[8]}$ antioxidant, ${ }^{[9]}$ anticancer, ${ }^{[10]}$ cytotoxic agents, ${ }^{[11]}$ antidyslipidemic agent, ${ }^{[12]}$ antileishmanial, ${ }^{[13]}$ anti-inflammatory agents. ${ }^{[14]}$

Diazo compounds showed many valuable medicinal and pharmaceutical activities such as inhibition of RNA, DNA and carcinogenesis, protein synthesis and nitrogen fixation. ${ }^{[15-18]}$

Bacterial and fungal resistance is one of the serious medical problems. Also, the levels rate of resistance is increasing to classical antibiotics. So, the development and discovery of effective antibacterial and antifungal drugs with novel mechanisms of action become urgent tasks for antimicrobial research programs. ${ }^{[19-22]}$

In light of these facts, the present study was designed to synthesize new chromene derivatives and evaluate their antimicrobial activity. As a trial to obtain novel class of antibacterial and antifungal agents, various groups were introduced into the target compounds

\section{RESULTS AND DISCUSSION \\ Chemistry}

Treatment salicylaldehyde derivative $\mathbf{1}^{[23]}$ with ethyl acetoacetate in boiling EtOH/DMF led to the formation of chromen-2-one derivative $\mathbf{2}$ as yellow solid in high yield (Scheme 1). Its IR spectrum showed absorption bands at: $1740,1650 \mathrm{~cm}^{-1}$ corresponding to $2 \mathrm{C}=0$ functional groups. ${ }^{1} \mathrm{H}$ NMR revealed signals at: $\delta=2.86$ and $8.87 \mathrm{ppm}$ corresponding to $\mathrm{CH}_{3}$ and chromene-H4, respectively. In

(c) B. BY This work is licensed under a Creative Commons Attribution 4.0 International License. 


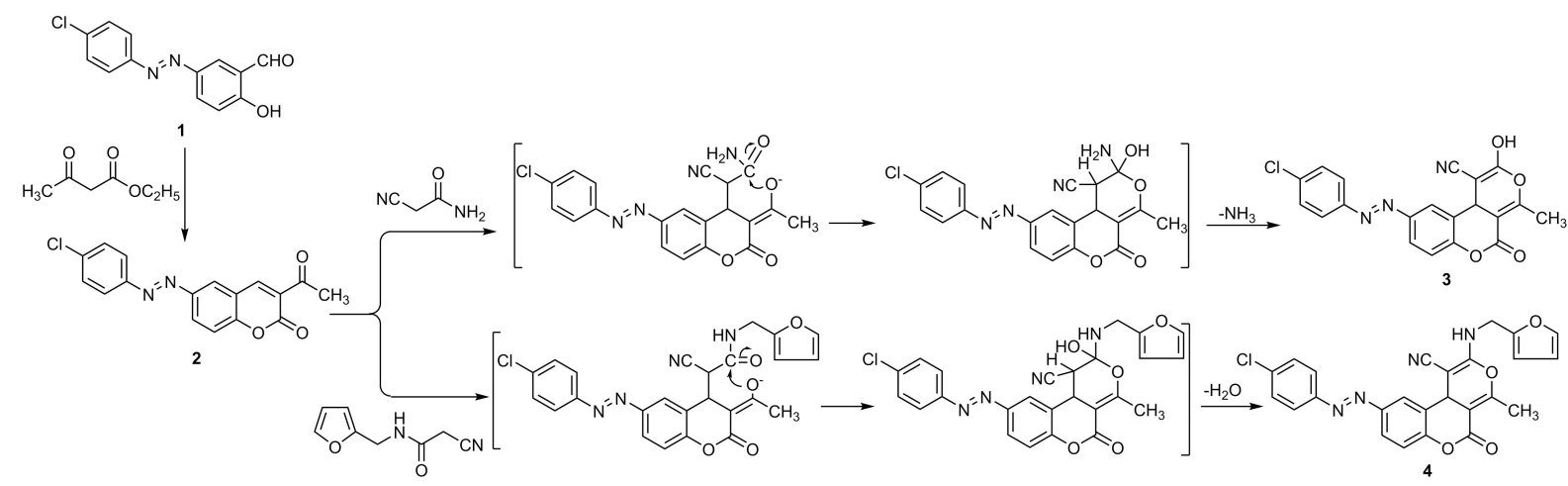

Scheme 1 . Synthesis of pyrano[3,4-c]chromene derivatives $\mathbf{3}$ and $\mathbf{4}$.

addition, the resulting chromene derivative was used for further chemical transformations as new routes for condensed chromene with possible biological activity. Acetylcoumarin derivative $\mathbf{2}$ was allowed to react with cyanoacetamide in presence of a catalytic amount of piperidine to give pyrano[3,4-c]chromene derivative 3. IR spectrum of compound 3 revealed absorption bands at: $\delta=$ 3413 and $2209 \mathrm{~cm}^{-1}$ for the $\mathrm{OH}$ and cyano functional groups. Its ${ }^{1} \mathrm{H}$ NMR spectrum showed two characterized signals at: $\delta=2.50$ and $\delta=9.04$ ppm assigned for the $\mathrm{CH}_{3}$ and $\mathrm{OH}$ protons.

Reaction of acetylcoumarin derivative 2 with 2 cyano- $N$-(furan-2-ylmethyl) acetamide ${ }^{[24]}$ in the presence of few drops of piperidine under reflux yielded the corresponding pyrano[3,4-c]chromene derivative 4. IR spectrum of $\mathbf{4}$ showed absorption bands at: 3253, 2209 and $1709 \mathrm{~cm}^{-1}$ corresponding to $\mathrm{NH}, \mathrm{C} \equiv \mathrm{N}$ and $\mathrm{C}=\mathrm{O}$ functional groups, respectively. ${ }^{1} \mathrm{H}$ NMR spectrum showed three signals at: $\delta=2.57,3.9$ and $5.07 \mathrm{ppm}$ due to $\mathrm{CH}_{3}, \mathrm{CH}_{2}$, and $\mathrm{CH}$ protons, respectively. Formation of $\mathbf{4}$ may be proceed through the addition of methylene group to the activated double bond of chromene to give Michael adduct, which cyclized to the respective pyrano[3,4-c]chromene 4 as a final product via elimination of water molecule.

Interaction of $\mathbf{1}$ with malononitrile in the presence of piperidine, 3-cyano chromane derivative $\mathbf{5}$ was obtained in good yield (Scheme 2). Its IR spectrum showed two characterized absorption bands at: 3348 and $2210 \mathrm{~cm}^{-1}$ corresponding to $\mathrm{NH}$ and $\mathrm{C} \equiv \mathrm{N}$ functional groups, respectively. Also, its ${ }^{1} \mathrm{H}$ NMR assigned multiple signals in the region 7.54-8.25 for Ar-H with H-pyrane. In addition, interaction of compound $\mathbf{5}$ with malononitrile or ethyl acetoacetate in presence of catalytic amount piperidine afforded chromenylmalononitrile derivative $\mathbf{6}$ and chromenyl ethyl cyanoacetate derivative $\mathbf{7}$, respectively. Chromenylmalononitrile 6 can be prepared by treatment compound 1 with two moles of malononitrile. IR spectrum of 7 revealed the presence of cyano and carbonyl ester functional groups at: 2203 and $1737 \mathrm{~cm}^{-1}$, respectively. Its
${ }^{1} \mathrm{H}$ NMR spectrum displayed in addition to the aromatic proton signals, two doubles signals at: $\delta=3.93$ and 4.16 ppm integrated for two protons due to the two $\mathrm{CH}$ protons. Moreover, its ${ }^{13} \mathrm{C}$ NMR confirmed the structure.

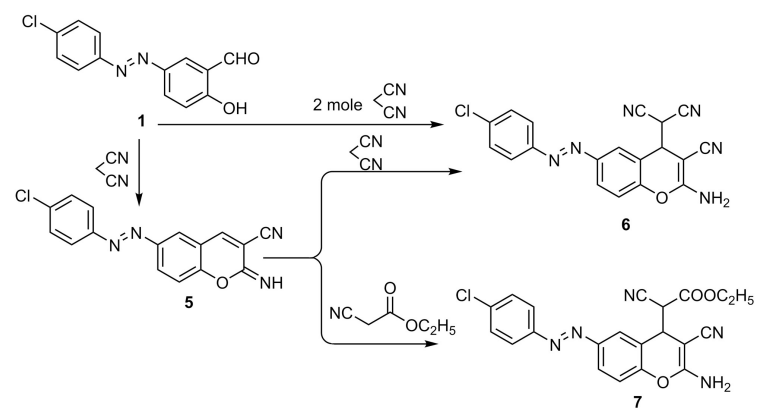

Scheme 2. Synthesis of chromene derivatives 5-7.

Cyclocondensation of the salicylaldehyde derivative 1 with diethyl malonate in presence of piperidine as catalyst afforded chromene-3-carboxylate derivative $\mathbf{8}$ as colored solid in high yield (Scheme 3). IR spectrum of 8 revealed absorption band at: $1722 \mathrm{~cm}^{-1}$ attributed for $\mathrm{C}=0$ functional group. ${ }^{1} \mathrm{H}$ NMR spectrum was characterized by the presence of triplet and quartet signals at: $\delta=1.20$ and $4.29 \mathrm{ppm}$ corresponding for ethyl protons. Iminochromene derivatives $\mathbf{9 a}$ and $\mathbf{9 b}$ were obtained through cyclocondensation of 1 with 2-cyano- $N$-(cyclohexyl)acetamide ${ }^{[25]}$ and 2-cyano- $N$-(furan-3-ylmethyl)acetamide, respectively. IR spectrum of 9a exhibited strong absorption bands at 3338 and $1672 \mathrm{~cm}^{-1}$ due to $\mathrm{NH}$ and $\mathrm{C}=\mathrm{O}$ functional groups, respectively. Its ${ }^{1} \mathrm{H}$ NMR showed signals at: $\delta=0.80-1.06$, 2.65-2.71, 8.96 and $10.17 \mathrm{ppm}$ corresponding to $\mathrm{CH}_{2}$ cyclohexyl, $\mathrm{CH}-\mathrm{N}$ cyclohexyl, $\mathrm{NH}$ and $\mathrm{CONH}$ protons, respectively.

On the same manner, interaction of 1 with 2-cyano$N^{\prime}$-(4-methoxybenzylidene)acetohydrazide afforded the corresponding iminochromene derivative 10. IR spectrum of 10 showed bands for amino and carbonyl groups at: 3301 and $1660 \mathrm{~cm}^{-1}$, respectively. ${ }^{1} \mathrm{H}$ NMR spectrum displayed 
two characterized singlet signals at: $\delta=3.80$ and $8.75 \mathrm{ppm}$ corresponding for $\mathrm{OCH}_{3}$ and azomethine protons, respectively. In addition, ${ }^{13} \mathrm{C}$ NMR showed three signals at: 55.8, 151.0 and 160.9 corresponding to $\mathrm{OCH}_{3}, \mathrm{C}=\mathrm{N}$ and $\mathrm{C}=\mathrm{O}$ carbons, respectively.

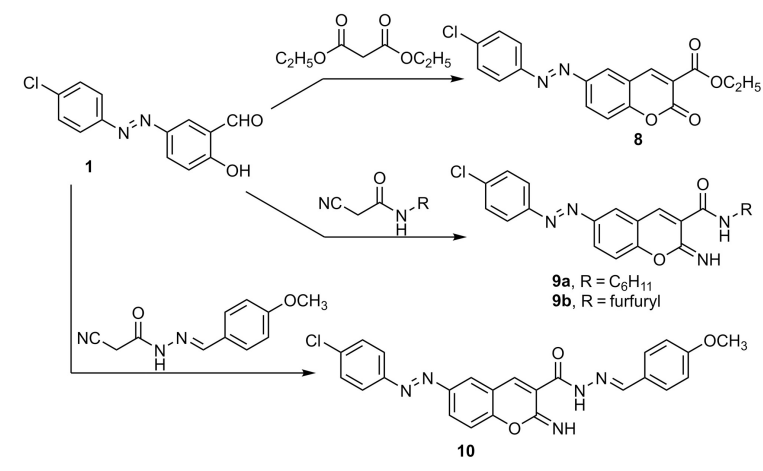

Scheme 3. Synthesis of chromene derivatives 8-10. methylene group to the activated double bond in compound $\mathbf{1 1}$ to afford the acyclic Michael adduct that cyclized, then aromatized to yield the final products.

Finally, 2-iminochromene derivative $\mathbf{9 b}$ was utilized as a starting material for further reaction. Hence, reaction of $\mathbf{9 b}$ with malononitrile or ethyl cyanoacetate in presence of a catalytic amount of piperidine afforded pyridino[3,4-c]chromene derivatives $\mathbf{1 4}$ and $\mathbf{1 5}$ (Scheme 5).

\section{Antimicrobial activity}

\section{ANTIBACTERIAL AND ANTIFUNGAL ACTIVITIES}

Fifteen test organisms representing three different microbial groups were used: Group 1: (Gram positive bacteria) Staphylococcus aureus (RCMB 010027), Staphylococcus epidermidis (RCMB 010024), Streptococcus pyogenes (RCMB 010015), and Bacillis subtilis (RCMB 010063); Group 2: (Gram negative bacteria) Proteous vulgaris (RCMB 010085), Klebsiella pneumonia (RCMB<smiles>CC(=O)CC(C)=O</smiles><smiles>CC(=O)c1c(C)[nH]c(=O)c2c(=N)oc3ccc(N=Nc4ccc(Cl)cc4)cc3c12</smiles><smiles>N#Cc1c(N)[nH]c(=O)c2c(=N)oc3ccc(N=Nc4ccc(Cl)cc4)cc3c12</smiles>

Scheme 4. Synthesis of pyridino[3,4-c]chromene derivatives 12 and 13.

In addition, chromene-3-carboxamide $\mathbf{1 1}$ was obtained via interaction of $\mathbf{1}$ with cyanoacetamide in $\mathrm{EtOH} /$ DMF containing catalytic amount of piperidine. ${ }^{1} \mathrm{H}$ NMR spectrum of 11 afforded two broad signals at: $\delta=8.62,9.04$ ppm attributed to $\mathrm{NH}$ and $\mathrm{NH}_{2}$ protons. Reaction of chromene derivative $\mathbf{1 1}$ with malononitrile or acetylacetone afforded products which formulated on the basis of elemental analysis and spectral data as chromenopyridine derivatives 12 and 13 , respectively (Scheme 4). ${ }^{1} \mathrm{H}$ NMR spectrum of $\mathbf{1 2}$ exhibited two broad signals at: $\delta=$ 4.31 and $9.54 \mathrm{ppm}$ due to $\mathrm{NH}_{2}$ and $\mathrm{NH}$ protons, respectively. ${ }^{1} \mathrm{H}$ NMR spectrum of 13 exhibited signals at: $\delta=$ 2.27, 2.47 and 8.70 ppm corresponding to $\mathrm{CH}_{3}, \mathrm{COCH}_{3}$ and $\mathrm{NH}$ protons, respectively. The formation of $\mathbf{1 2}$ and $\mathbf{1 3}$ is assumed through the Michael addition of the activated

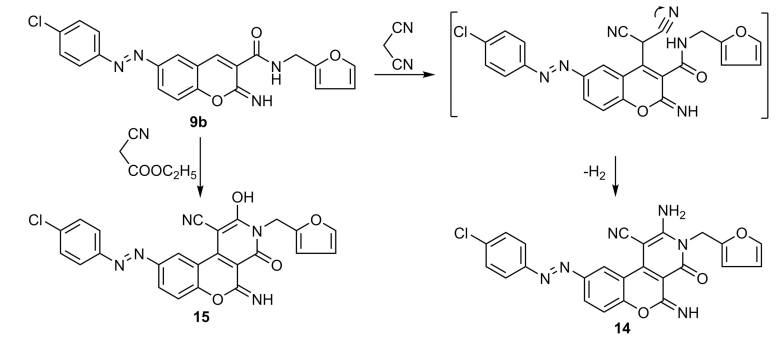

Scheme 5 . Synthesis of pyridino[3,4-c]chromene derivatives 14 and 15 .

010093), Shigella flexneri (RCMB 0100542) and Pseudomonas aeruginosa (RCMB 010043); Group 3: (Fungi) Aspergillus fumigates (RCMB 02564), Aspergillus clavatus 
(RCMB02593), Candida albicans (RCMB 05035), and Penicillium marneffei (RCMB 01267). Agar-diffusion method[26] was used for the determination of the preliminary screening of antibacterial activity. The newly synthesized target compounds were evaluated against clinical isolates of standard strains of fungi by the broth dilution method according to NCCLs ${ }^{[27]}$ Ampicillin, Gentamycin and Amphotericin B were used as reference drugs for Gram-positive bacteria, Gram-negative bacteria and fungi, respectively. The results were recorded for each tested compounds as the average diameter of inhibition zones of bacterial growth around the discs in $\mathrm{mm}$. The inhibition zone diameters, attributed to the tested original concentration (5 $\mathrm{mg} / \mathrm{mL}$ ) as a preliminary test, are shown in Table 1.

Concerning the antimicrobial activity of chromene derivatives $\mathbf{2}, \mathbf{5}, \mathbf{8}, \mathbf{9 a}, \mathbf{b}, \mathbf{1 0}, \mathbf{1 1}$, the results displayed those derivatives showed moderate to good activities against most of all the screened organisms. Using the general structure provided in Figure 1 , certain aspects of the structure activity relationships for these compounds can be more clearly highlighted.

The results revealed that compound $\mathbf{2}$ showed antimicrobial activity nearly equipotent to the reference drug against all screened organisms. While compound $\mathbf{8}$ showed antimicrobial activity nearly equipotent to the reference drug only against gram -ve bacteria. Chromen-2- one $\mathbf{5}, 9 \mathrm{9a}, \mathrm{b}, 10$ and $\mathbf{1 1} \mathrm{X}$ was $\mathrm{NH}$ group and $\mathrm{R}$ were cyano or carboxamide derivatives. Regarding the effect of $R$ group, compound $\mathbf{5}$ showed high antimicrobial activity against all screened organisms. Compound $\mathbf{9 b}$ and 10 showed good antimicrobial activities only against gram +ve bacteria. Compound $\mathbf{1 1}$ showed high antimicrobial activity only against gram -ve bacteria. Compound 9a showed high antimicrobial activity only against fungi. Using the general structure provided in Figure 1, compound 6 showed high antimicrobial activity against all screened bacteria while compound $\mathbf{7}$ showed good activity only against fungi.

Using the general structure provided in Figure 2, pyrano[3,4-c]chromene derivatives 3 and $4 ; \mathrm{R}$ were hydroxyl group (3) or $\mathrm{NHCH}_{2}$ furfuryl moiety (4). Regarding the effect of $\mathrm{R}$ group, pyrano[3,4-c]chromene $\mathbf{3}$ showed high antimicrobial activity against all screened bacteria. While, pyrano[3,4-c]chromene 4 showed moderate activity against most of tested organisms.

Concerning the antimicrobial activity of pyridino[3,4-c]chromene derivatives 12-15: The presence of pyridino[3,4c]chromene moiety resulted in the highest antimicrobial activity among all the compounds investigated in this study. The presence of pyridino[3,4-c]-chromene moiety exhibited the highest antibacterial activity against most of the organisms; pyridino[3,4-c]-chromene moiety showed results greater than the reference drug against most of the

Table 1. Antimicrobial activities of the synthesized compounds against the pathological Gram +ve bacteria, Gram -ve bacteria and fungi expressed as inhibition diameter zones in millimeters

\begin{tabular}{|c|c|c|c|c|c|c|c|c|c|c|c|c|}
\hline \multirow{2}{*}{$\begin{array}{c}\text { Compd. } \\
\text { No. }\end{array}$} & \multicolumn{4}{|c|}{ Gram +ve bacteria } & \multicolumn{4}{|c|}{ Gram -ve bacteria } & \multicolumn{4}{|c|}{ Fungi } \\
\hline & S. aureus & S. epider. & S. pyoge. & B. subtili. & P. vulga. & K. pneu. & S. Flexn. & P. aerug. & A. fumig. & A. clavat. & C. albic. & P. neffei \\
\hline 2 & $24.5 \pm 0.3$ & $22.5 \pm 0.4$ & $23.7 \pm 0.8$ & $26.3 \pm 0.6$ & $22.6 \pm 0.2$ & $21.0 \pm 0.6$ & $21.4 \pm 0.6$ & $16.3 \pm 0.5$ & $21.2 \pm 0.3$ & $19.2 \pm 0.2$ & $18.7 \pm 0.3$ & $20.7 \pm 0.5$ \\
\hline 3 & $25.6 \pm 0.5$ & $22.4 \pm 0.5$ & $21.4 \pm 0.2$ & $28.6 \pm 0.5$ & $20.2 \pm 0.3$ & $23.4 \pm 0.5$ & $22.4 \pm 0.3$ & $15.0 \pm 0.2$ & $19.5 \pm 0.3$ & $18.8 \pm 0.3$ & $16.5 \pm 0.4$ & $19.8 \pm 0.3$ \\
\hline 4 & $15.1 \pm 0.7$ & $14.5 \pm 0.5$ & NA & $13.1 \pm 0.3$ & $12.3 \pm 0.2$ & $13.5 \pm 0.5$ & NA & NA & $14.5 \pm 0.6$ & $11.7 \pm 0.5$ & $10.3 \pm 0.3$ & $13.5 \pm 0.8$ \\
\hline 5 & $24 \pm 0.5$ & $20.9 \pm 0.6$ & $18.9 \pm 0.3$ & $24.8 \pm 0.2$ & $21.2 \pm 0.7$ & $22.8 \pm 0.2$ & $21.6 \pm 0.5$ & $16.8 \pm 0.6$ & $20.4 \pm 0.6$ & $18.9 \pm 0.3$ & $21.9 \pm 0.6$ & $20.2 \pm 0.3$ \\
\hline 6 & $15.3 \pm 0.4$ & $14.7 \pm 0.4$ & NA & $17.2 \pm 0.2$ & $13.2 \pm 0.3$ & $15.2 \pm 0.3$ & $12.5 \pm 0.5$ & NA & $21.1 \pm 0.3$ & $19.1 \pm 0.2$ & $23.8 \pm 0.4$ & $18.9 \pm 0.3$ \\
\hline 7 & $27.8 \pm 0.2$ & $23.2 \pm 0.5$ & $23.2 \pm 0.3$ & $27.9 \pm 0.4$ & $21.2 \pm 0.7$ & $23.8 \pm 0.3$ & $22.9 \pm 0.3$ & $16.6 \pm 0.4$ & $14.2 \pm 0.5$ & $12.6 \pm 0.2$ & NA & $13.5 \pm 0.6$ \\
\hline 8 & $17.2 \pm 0.4$ & $14.2 \pm 0.2$ & NA & $18.4 \pm 0.1$ & $22.5 \pm 0.3$ & $24.3 \pm 0.1$ & $22.4 \pm 0.3$ & $16.9 \pm 0.3$ & $13.5 \pm 0.5$ & $11.8 \pm 0.4$ & $10.9 \pm 0.3$ & $12.2 \pm 0.6$ \\
\hline $9 a$ & $15.2 \pm 0.2$ & $16.9 \pm 0.3$ & NA & $17.1 \pm 0.2$ & $12.5 \pm 0.5$ & $13.9 \pm 0.4$ & $11.9 \pm 0.3$ & NA & $21.8 \pm 0.3$ & $19.2 \pm 0.3$ & $22.5 \pm 0.4$ & $19.9 \pm 0.6$ \\
\hline $9 b$ & $26.7 \pm 0.6$ & $22.0 \pm 0.2$ & $21.7 \pm 0.3$ & $28.8 \pm 0.3$ & $14.8 \pm 0.4$ & $15.5 \pm 0.7$ & $14.7 \pm 0.4$ & NA & $14.2 \pm 0.3$ & $15.1 \pm 0.3$ & NA & $13.4 \pm 0.1$ \\
\hline 10 & $24.3 \pm 0.5$ & $23.3 \pm 0.5$ & $24.2 \pm 0.3$ & $28.3 \pm 0.5$ & $14.2 \pm 0.6$ & $12.6 \pm 0.4$ & $11.5 \pm 0.6$ & NA & $13.4 \pm 0.6$ & $11.7 \pm 0.5$ & NA & $16.8 \pm 0.2$ \\
\hline 11 & $17.7 \pm 0.3$ & $14.4 \pm 0.2$ & NA & $16.5 \pm 0.4$ & $21.5 \pm 0.2$ & $23.3 \pm 0.6$ & $21.4 \pm 0.6$ & $15.2 \pm 0.5$ & $19.9 \pm 0.4$ & $20.0 \pm 0.4$ & $23.0 \pm 0.2$ & $20.2 \pm 0.5$ \\
\hline 12 & $29.2 \pm 0.3$ & $26.1 \pm 0.3$ & $28.3 \pm 0.4$ & $36.3 \pm 0.4$ & $24.5 \pm 0.2$ & $27.7 \pm 0.3$ & $25.2 \pm 0.6$ & $18.2 \pm 0.4$ & $25.1 \pm 0.6$ & $24.1 \pm 0.3$ & $26.9 \pm 0.4$ & $26.4 \pm 0.5$ \\
\hline 13 & $30.0 \pm 0.5$ & $28.2 \pm 0.5$ & $28.5 \pm 0.3$ & $32.9 \pm 0.3$ & $23.8 \pm 0.2$ & $28.2 \pm 0.3$ & $25.5 \pm 0.3$ & $19.4 \pm 0.4$ & $24.8 \pm 0.6$ & $22.5 \pm 0.6$ & $27.3 \pm 0.6$ & $23.6 \pm 0.5$ \\
\hline 14 & $29.9 \pm 0.4$ & $27.6 \pm 0.4$ & $28.5 \pm 0.4$ & $34.8 \pm 0.3$ & $25.1 \pm 0.5$ & $27.2 \pm 0.1$ & $26.2 \pm 0.3$ & $18.8 \pm 0.3$ & $23.6 \pm 0.4$ & $23.9 \pm 0.5$ & $28.4 \pm 0.8$ & $25.2 \pm 0.7$ \\
\hline 15 & $29.5 \pm 0.7$ & $26.8 \pm 0.4$ & $27.5 \pm 0.4$ & $35.7 \pm 0.7$ & $25.9 \pm 0.1$ & $29.2 \pm 0.2$ & $26.2 \pm 0.6$ & $17.8 \pm 0.4$ & $23.9 \pm 0.3$ & $22.7 \pm 0.2$ & $28.4 \pm 0.2$ & $24.9 \pm 0.4$ \\
\hline Ampicillin & $28.9 \pm 1$ & $25.4 \pm 0.1$ & $26.4 \pm 0.3$ & $34.6 \pm 0.3$ & ---- & ---- & $-\cdots--$ & --- & ----- & ---- & ---- & ---- \\
\hline Gentamycin & ---- & ---- & ---- & ---- & $23.4 \pm 0.3$ & $26.3 \pm 0.1$ & $24.8 \pm 0.2$ & $17.3 \pm 0.1$ & ----- & ---- & ---- & ----- \\
\hline Amphotericin B & -- & ---- & ---- & ---- & ---- & --- & ---. & ---- & $23.7 \pm 0.1$ & $21.9 \pm 0.1$ & $26.4 \pm 0.2$ & $22.6 \pm 0.3$ \\
\hline
\end{tabular}




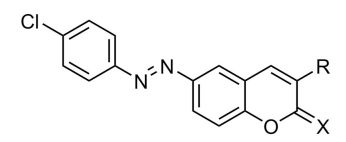

2; $\mathrm{X}=\mathrm{O}, \mathrm{R}=\mathrm{COCH}_{3}$

$5 ; X=\mathrm{NH}, \mathrm{R}=\mathrm{CN}$

8; $X=O, R=$ COOEt

$9 \mathrm{a} ; \mathrm{X}=\mathrm{NH}, \mathrm{R}=\mathrm{CONHC}_{6} \mathrm{H}_{11}$

10; $X=\mathrm{NH}, \mathrm{R}=\mathrm{CONHN}=\mathrm{CHC}_{6} \mathrm{H}_{4}\left(\mathrm{OCH}_{3}\right)^{-4}$

11; $X=\mathrm{NH}, \mathrm{R}=\mathrm{CONH}_{2}$

Figure 1.

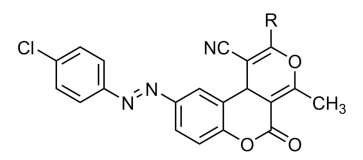

3; $\mathrm{R}=\mathrm{OH}$

4; $\mathrm{R}=\mathrm{NHCH}_{2}$ furfuryl<smiles>[X]C(C)C1C(N)=C(N)Oc2ccc(NNc3ccc(Cl)cc3)cc21</smiles>
$6 ; \mathrm{X}=\mathrm{CN}$ $7 ; \mathrm{X}=\mathrm{COOC}_{2} \mathrm{H}_{5}$
Figure 2.

organisms. Using the general structure provided in Figure 2 , a moderate difference in antimicrobial activity is noted between the tested pyridino[3,4-c]chromene derivatives, this indicate that the main effect related to the presence of the pyridino[3,4-c]chromene moiety. The comparison between the antimicrobial activity of pyridino[3,4-c]chromene and standard reference drugs against the used Gram positive, Gram negative bacteria and fungi is represented graphically in Figure 3.

\section{MINIMUM INHIBITORY CONCENTRATIONS AGAINST GRAM POSITIVE BACTERIA, GRAM NEGATIVE BACTERIA AND FUNGI}

The minimal inhibitory concentrations (MICs) for the promising compounds were determined using twofold serial dilution method ${ }^{[27]} \mathrm{MICs}$ results $(\mu \mathrm{g} / \mathrm{mL})$ of the most promising derivatives $\mathbf{2}, \mathbf{3}, \mathbf{5}, \mathbf{6}, \mathbf{9 a}$, and 11-15 are presented in Table 2. The majority of synthesized compounds showed varying degrees of inhibition against the test panel of species.

Pyridino[3,4-c]chromene 14 showed equipotent potency of standard drugs in inhibiting the growth of S. pyogenes (MIC $0.24 \mu \mathrm{g} / \mathrm{mL}$ ), P. vulgaris (MIC $1.95 \mu \mathrm{g} / \mathrm{mL}$ ), S. flexneri (MIC $0.48 \mu \mathrm{g} / \mathrm{mL}$ ), A. clavatus (MIC $1.95 \mu \mathrm{g} / \mathrm{mL}$ ) and P. Marneffei (MIC $1.95 \mu \mathrm{g} / \mathrm{mL}$ ). Pyridino[3,4-c]chromene 14 displayed $50 \%$ less activity compared to standard drugs against $P$. aeruginosa (MIC $62.5 \mu \mathrm{g} / \mathrm{mL}$ ).

Chromen-2-one 11 showed equipotent potency of standard drugs in inhibiting the growth of S. flexneri (MIC $0.48 \mu \mathrm{g} / \mathrm{mL}$ ), P. aeruginosa (MIC $31.25 \mu \mathrm{g} / \mathrm{mL}$ ) and P. Marneffei (MIC $1.95 \mu \mathrm{g} / \mathrm{mL}$ ). Chromen-2-one 11 displayed $50 \%$ less activity compared to standard drugs against S. pyogenes (MIC $0.48 \mu \mathrm{g} / \mathrm{mL}$ ), P. vulgaris (MIC 3.9 $\mu \mathrm{g} / \mathrm{mL}$ ), A. Fumigates (MIC $1.95 \mu \mathrm{g} / \mathrm{mL}$ ), A. clavatus (MIC $3.9 \mu \mathrm{g} / \mathrm{mL}$ ) and C. albicans (MIC $0.48 \mu \mathrm{g} / \mathrm{mL}$ ).

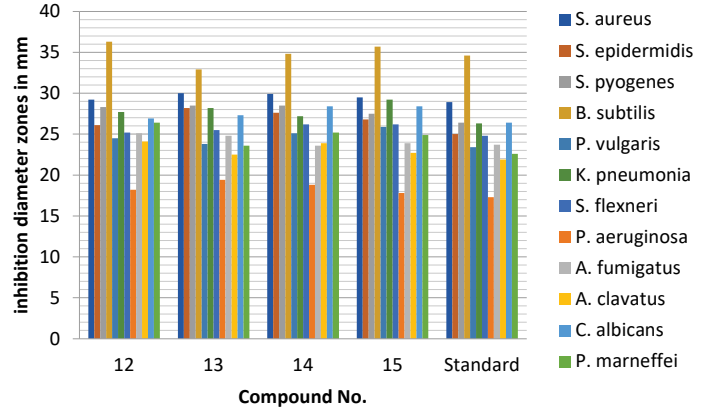

Figure 3. The comparison between the antimicrobial activities of pyridino[3,4-c]chromene and standard drug against the used Gram +ve, Gram -ve bacteria and fungi.

Pyridino[3,4-c]chromene 12 showed equipotent potency of standard drugs in inhibiting the growth of S. pyogenes (MIC $0.24 \mu \mathrm{g} / \mathrm{mL}$ ) and P. vulgaris (MIC 1.95 $\mu \mathrm{g} / \mathrm{mL}$ ). Pyridino[3,4-c]chromene 12 displayed $50 \%$ less activity compared to standard drugs against $S$. epidermidis (MIC $0.97 \mu \mathrm{g} / \mathrm{mL}$ ), K. pneumonia (MIC $0.48 \mu \mathrm{g} / \mathrm{mL}$ ), P. aeruginosa (MIC $62.5 \mu \mathrm{g} / \mathrm{mL}$ ), A. Fumigates (MIC 1.95 $\mu \mathrm{g} / \mathrm{mL}$ ), A. clavatus (MIC $3.9 \mu \mathrm{g} / \mathrm{mL}$ ) and P. Marneffei (MIC $3.9 \mu \mathrm{g} / \mathrm{mL}$ ). Pyridino[3,4-c]chromene 13 showed equipotent potency of standard drugs in inhibiting the growth of A. Fumigates (MIC $0.97 \mu \mathrm{g} / \mathrm{mL}$ ) and P. Marneffei (MIC 1.95 $\mu \mathrm{g} / \mathrm{mL}$ ). pyridino[3,4-c]chromene $\mathbf{1 3}$ displayed $50 \%$ less activity compared to standard drugs against $C$. albicans (MIC $0.48 \mu \mathrm{g} / \mathrm{mL}$ ).

Chromen-2-one 9a showed equipotent potency of standard drugs in inhibiting the growth of $A$. fumigates (MIC $0.97 \mu \mathrm{g} / \mathrm{mL}$ ). Chromen-2-one derivative 9a displayed $50 \%$ less activity compared to standard drugs against S. pyogenes (MIC $0.48 \mu \mathrm{g} / \mathrm{mL}$ ), P. vulgaris (MIC $3.9 \mu \mathrm{g} / \mathrm{mL}$ ), $P$. aeruginosa (MIC $62.5 \mu \mathrm{g} / \mathrm{mL}$ ), A. clavatus (MIC $3.9 \mu \mathrm{g} / \mathrm{mL}$ ), C. albicans (MIC $0.48 \mu \mathrm{g} / \mathrm{mL}$ ) and P. Marneffei (MIC $3.9 \mu \mathrm{g} / \mathrm{mL}$ ).

Chromene 5 displayed $50 \%$ less activity compared to standard drugs against S. epidermidis (MIC $0.24 \mu \mathrm{g} / \mathrm{mL}$ ), P. vulgaris (MIC $3.9 \mu \mathrm{g} / \mathrm{mL}$ ), K. pneumonia (MIC $0.48 \mu \mathrm{g} / \mathrm{mL}$ ), A. clavatus (MIC $3.9 \mu \mathrm{g} / \mathrm{mL}$ ) and P. Marneffei (MIC $3.9 \mu \mathrm{g} / \mathrm{mL}$ ).

Chromene $\mathbf{2}$ displayed $50 \%$ less activity compared to standard drugs against $P$. vulgaris (MIC $3.9 \mu \mathrm{g} / \mathrm{mL}$ ) and $P$. Marneffei (MIC $3.9 \mu \mathrm{g} / \mathrm{mL}$ ).T2

\section{CONCLUSION}

Chromen-2-one, pyrano[3,4-c]chromene and pyridino[3,4-c]chromene derivatives bearing a diazo moiety were synthesized in order to evaluate their antibacterial and antifungal activities. Regarding the effect of each derivative against bacterial and fungal strains, results of antimicrobial activity in this study revealed that: the majority of these 
Table 2. Minimum inhibitory concentration $(\mu \mathrm{g} / \mathrm{mL})$ of the most potent synthesized compounds against the pathological organisms

\begin{tabular}{|c|c|c|c|c|c|c|c|c|c|c|c|c|}
\hline Compd. No. & S. aureus & S. epidermidis & S. pyogenes & B. subtilis & P. vulgaris & K. pneumonia & S. flexneri & P. aeruginosa & A. fumigatus & A. clavatus & C. albicans & P. marneffei \\
\hline 2 & 0.97 & 15.63 & 3.9 & 1.95 & 3.9 & 1.95 & 3.9 & 125 & 3.9 & 15.63 & 31.25 & 3.9 \\
\hline 3 & 3.9 & 3.9 & 31.25 & 1.95 & 7.81 & 3.9 & 7.81 & 250 & 3.9 & 31.25 & 62.5 & 7.81 \\
\hline 5 & 0.24 & 0.24 & 3.9 & 0.06 & 3.9 & 0.48 & 3.9 & 500 & 15.63 & 3.9 & 31.25 & 3.9 \\
\hline 6 & 3.9 & 1.95 & 62.5 & 0.97 & 7.81 & 1.95 & 7.81 & 250 & 7.81 & 15.63 & 31.25 & 15.63 \\
\hline $9 a$ & 0.24 & 1.95 & 0.48 & 0.06 & 3.9 & 0.84 & 1.95 & 62.5 & 0.97 & 3.9 & 0.48 & 3.9 \\
\hline 11 & 0.97 & 1.95 & 0.48 & 0.06 & 3.9 & 1.95 & 0.48 & 31.25 & 1.95 & 3.9 & 0.48 & 1.95 \\
\hline 12 & 0.24 & 0.97 & 0.24 & 0.06 & 1.95 & 0.48 & 3.9 & 62.5 & 1.95 & 3.9 & 0.97 & 3.9 \\
\hline 13 & 3.9 & 62.5 & 125 & 62.5 & 7.81 & 1.95 & 3.9 & 250 & 0.97 & 7.81 & 0.48 & 1.95 \\
\hline 14 & 0.24 & 3.9 & 0.24 & 0.06 & 1.95 & 0.97 & 0.48 & 62.5 & 0.24 & 1.95 & 0.97 & 1.95 \\
\hline 15 & 3.9 & 62.5 & 31.25 & 125 & 7.81 & 3.9 & 15.63 & 250 & 62.5 & 7.81 & 62.5 & 125 \\
\hline Ampicillin & 0.06 & 0.48 & 0.24 & 0.007 & ----- & ---- & ---- & ---- & ---- & ---- & $-\cdots--$ & ---- \\
\hline Gentamycin & ---- & ----- & ---- & ---- & 1.95 & 0.24 & 0.48 & 31.25 & ---- & ---- & ---- & ----- \\
\hline Amphotericin B & & -.--- & -.--- & ----- & ----. & ----- & .---- & -.--- & 0.97 & 1.95 & 0.24 & 1.95 \\
\hline
\end{tabular}

compounds showed potent antibacterial and antifungal activities against the tested strains of bacteria and fungi. Pyridino[3,4-c]chromene moiety resulted in the highest antimicrobial activity among all the compounds investigated in this study. Pyridino[3,4-c]chromene moiety showed results greater than the reference drug against most of the organisms.

\section{Experimental Section}

All melting points are recorded on digital Gallen Kamp MFB595 instrument and may be uncorrected. The IR spectra $(\mathrm{KBr})\left(\mathrm{cm}^{-1}\right)$ were measured on a JASCO spectrophotometer. ${ }^{1} \mathrm{H}$ NMR spectra were recorded on Bruker spectrometers (at $500 \mathrm{MHz}$ ) and are reported relative to deuterated solvent signals in deuterated dimethylsulfoxide (DMSO- $\left.d_{6}\right) .{ }^{13} \mathrm{C}$ NMR spectra were recorded on Bruker Spectrometers (at $125 \mathrm{MHz}$ ) in deuterated dimethylsulfoxide (DMSO- $d_{6}$ ). The purity of the synthesized compounds was monitored by TLC. Elemental analyses were carried out by the Microanalytical Research Center, Faculty of Science, Cairo University. Analytical results for $\mathrm{C}, \mathrm{H}$ and $\mathrm{N}$ were within $-/+0.4$ of the calculated values. The antimicrobial screening and minimal inhibitory concentrations of the tested compounds were carried out at the Regional Center for Mycology and Biotechnology, Al-Azhar University, Cairo, Egypt.

\section{SYNTHESIS OF 3-ACETYL-6-((4-CHLOROPHENYL)- DIAZENYL)-2H-CHROMEN-2-ONE (2)}

Equimolar amounts of compound $\mathbf{1}(0.01 \mathrm{~mol})$ and ethyl acetoacetate $(0.01 \mathrm{~mol})$ in a mixture of $15 \mathrm{~mL} \mathrm{EtOH}$ and 5 $\mathrm{mL}$ DMF was treated with few drops of piperidine. The reaction mixture was heated under reflux for $4 \mathrm{~h}$, and then allowed to cool. The precipitate that formed was filtered, washed with ethanol, dried and crystallized from ethanol to give compound $\mathbf{2}$ as yellow crystals. Yield $85 \%$; m.p. 235-237 ${ }^{\circ} \mathrm{C}$; IR: $v / \mathrm{cm}^{-1}=1740,1650(2 \mathrm{C}=0) ;{ }^{1} \mathrm{H}$ NMR: $\delta / \mathrm{ppm}=2.86$ (s, $\left.3 \mathrm{H}, \mathrm{CH}_{3}\right), 7.58-8.25$ (m, 7H, Ar-H), 8.87 (s, $1 \mathrm{H}, \mathrm{CH}$-pyrane); ${ }^{13} \mathrm{C}$ NMR: $29.3\left(\mathrm{CH}_{3}\right), 118.0,119.5,122.2,123.6,124.4$ (2C), 129.3 (2C), 131.0, 136.1, 138.4, 150.2, 150.8, 155.7, 159.4, 188.7 $(\mathrm{C}=0) ; \mathrm{MS}: \mathrm{m} / \mathrm{z}=326\left(\mathrm{M}^{+} ; 3.9\right), 170(5.5), 153(7.0), 127$ (70.5), 65 (77), 55 (100); Anal. Calcd. For $\mathrm{C}_{17} \mathrm{H}_{11} \mathrm{ClN}_{2} \mathrm{O}_{3}$ (326.74): C, 62.49; H, 3.39; N, 8.57; found: C, 62.65; H, 3.24; N, 8.87

\section{SYNTHESIS OF 9-((4-CHLOROPHENYL)DIAZENYL)-2- HYDROXY-4-METHYL-5-OXO-5,10b-DIHYDRO- PYRANO[3,4-c]CHROMENE-1-CARBONITRILE (3)}

Cyanoacetamide $(0.01 \mathrm{~mol})$ was added to a solution of compound 2 (0.01 mol) in mixture of $15 \mathrm{~mL} \mathrm{EtOH,} 5 \mathrm{~mL}$ DMF and 3 drops of piperidine. The reaction mixture was heated under reflux for 4 hours and concentrated to its halfvolume. The solid product was filtered off and recrystallized from ethanol to give compound $\mathbf{3}$ as pale yellow crystals. Yield $70 \%$; m.p. $265-267^{\circ} \mathrm{C}$; IR: $v / \mathrm{cm}^{-1}=3413$ (broad, OH), 2925 (CH-aliphatic), $2209(\mathrm{C} \equiv \mathrm{N}), 1671(\mathrm{C}=\mathrm{O}) ;{ }^{1} \mathrm{H}$ NMR: $\delta / p p m=2.5\left(\mathrm{~s}, 3 \mathrm{H}, \mathrm{CH}_{3}\right), 5.06$ (s, 1H, H-pyrane), 7.59-8.22 (m, 7H, Ar-H), 9.04 (br, 1H, OH); ${ }^{13} \mathrm{C} \mathrm{NMR:} 18.0\left(\mathrm{CH}_{3}\right), 52.1$, $106.7,117.4,121.4,121.8,124.4,124.6,125.0,128.6$, 129.1, 130.2 (2C), 136.5, 149.4, 151.2, 152.8, 159.0, 162.3, 185.2; Anal. Calc.: $\mathrm{C}_{20} \mathrm{H}_{12} \mathrm{ClN}_{3} \mathrm{O}_{4}$ : C, 61.00; $\mathrm{H}, 3.07 ; \mathrm{N}, 10.67$; Found: C, 60.89; H, 3.34; N, 10.97 .

SYNTHESIS OF 9-((4-CHLOROPHENYL)DIAZENYL)-2(FURAN-2-YLMETHYLAMINO)-4-METHYL-5-OXO-5,10bDIHYDROPYRANO[3,4-C]CHROMENE-1-CARBONITRILE (4) A mixture of 2 (0.01 mol) and 2-cyano- $N$-(furan-2-ylmethyl) acetamide $(0.01 \mathrm{~mol})$ in a mixture of $15 \mathrm{~mL}$ EtOH and $5 \mathrm{~mL}$ DMF containing a catalytic amount of piperidine was heated under reflux for $4 \mathrm{~h}$, then left to cool. The solid product formed was filtrated off, washed with $\mathrm{EtOH}$, dried, 
and finally crystallized from EtOH/DMF to give compound $\mathbf{4}$ as pale yellow crystals. Yield $60 \%$; m.p. 290-292 ${ }^{\circ} \mathrm{C}$; IR: $v / \mathrm{cm}^{-1}=3253(\mathrm{NH}), 2925$ (aliphatic $\mathrm{CH}$ ), $2209(\mathrm{C} \equiv \mathrm{N}), 1709$ ( $\mathrm{C}=0) ;{ }^{1} \mathrm{H}$ NMR: $\delta / \mathrm{ppm}=2.57\left(\mathrm{~s}, 3 \mathrm{H}, \mathrm{CH}_{3}\right), 3.9\left(\mathrm{~s}, 2 \mathrm{H}, \mathrm{CH}_{2}\right)$, 5.07 (s, 1H, H-4-pyrane), 5.15 (d, 1H, CH-furfuryl), 6.40 (d, $1 \mathrm{H}, \mathrm{CH}$-furfuryl), 7.50-8.56 (m, 8H, 7Ar-H + CHO-furfuryl), $9.6\left(\mathrm{br}, 1 \mathrm{H}, \mathrm{NH}\right.$ cancelled with $\left.\mathrm{D}_{2} \mathrm{O}\right) ;{ }^{13} \mathrm{C} \mathrm{NMR}: 17.3\left(\mathrm{CH}_{3}\right)$, $37.7\left(\mathrm{CH}_{2}\right), 45.2(\mathrm{CH}), 106.0,110.6,111.4,119.1,120.4$, 121.8, 124.4 (2C), 124.9, 128.6 (2C), 129.1 (2C), 136.8, 142.3, 145.8, 150.2, 151.2, 152.5, 159.3, 161.9, 176.6; Anal. Calc: $\mathrm{C}_{25} \mathrm{H}_{17} \mathrm{ClN}_{4} \mathrm{O}_{4}$ (472.89): C, 63.50; $\mathrm{H}, 3.62 ; \mathrm{N}, 11.85$; Found: $\mathrm{C}, 63.36 ; \mathrm{H}, 3.66 ; \mathrm{N}, 11.22$.

\section{SYNTHESIS OF 6-((4-CHLOROPHENYL)DIAZENYL)-2- IMINO-2H-CHROMENE-3-CARBONITRILE (5)}

A mixture of compound 1 (0.01 mol), malononitrile (0.01 $\mathrm{mol}$ ) and drops of piperidine in a mixture of $15 \mathrm{~mL}$ EtOH and $5 \mathrm{~mL}$ DMF was heated under reflux for 2 hours. The solid product formed on hot collected by filtration and recrystallized from ethanol/DMF to give compound $\mathbf{5}$ as brown crystals. Yield $80 \%$; m.p. $>300{ }^{\circ} \mathrm{C}$; IR: $v / \mathrm{cm}^{-1}=3348(\mathrm{NH})$, $2210(\mathrm{C} \equiv \mathrm{N}) ;{ }^{1} \mathrm{H}$ NMR: $\delta / \mathrm{ppm}=7.54-7.78(\mathrm{~m}, 3 \mathrm{H}, \mathrm{Ar}-\mathrm{H})$, 7.87-8.25 (m, 5H, Ar-H \& H-pyrane), $9.53(\mathrm{br}, 1 \mathrm{H}, \mathrm{NH}$ exchangeable with $\left.\mathrm{D}_{2} \mathrm{O}\right) ;{ }^{13} \mathrm{C}$ NMR: $110.3,112.7,115.4$, $116.8,117.5,121.5,123.6,123.7,124.4,129.1,136.5,145.2$, 150.8, 156.5, 164.4; Anal. Calc. $\mathrm{C}_{16} \mathrm{H}_{9} \mathrm{ClN}_{4} \mathrm{O}$ (308.73): C, 62.25; H, 2.94; N, 18.15; Found: C, 62.43; H, 2.65; N, 18.22.

SYNTHESIS OF 2-(6-((4-CHLOROPHENYL)DIAZENYL)-3CYANO-2-IMINO-2H-CHROMEN-4-YL)MALONONITRILE (6) Method A: A mixture of compound 1 (0.01 mol), malononitrile $(0.02 \mathrm{~mol})$ in a mixture of $15 \mathrm{~mL} E \mathrm{EtOH}, 5 \mathrm{~mL}$ DMF and 3 drops of piperidine was heated under refluxed for $3 \mathrm{~h}$. The solid product formed on hot, collected by filtration and recrystallized from ethanol as orange crystals. Yield $82 \%$. Method B: A mixture of compound 5 (0.01 mol), malononitrile $(0.01 \mathrm{~mol})$ in a mixture of $15 \mathrm{~mL} E \mathrm{EtOH}, 5 \mathrm{~mL}$ DMF and 3 drops of piperidine was heated under refluxed for $2 \mathrm{~h}$. The solid product formed on hot, collected by filtration and recrystallized from ethanol as orange crystals. Yield $79 \%$; m.p. $>300{ }^{\circ} \mathrm{C}$; IR: v/cm ${ }^{-1}=3335\left(\mathrm{NH}_{2}\right), 2209$ $(\mathrm{C} \equiv \mathrm{N}) ;{ }^{1} \mathrm{H}$ NMR: $\delta / \mathrm{ppm}=3.76(\mathrm{~d}, 1 \mathrm{H}, J=3.7 \mathrm{~Hz}, \mathrm{CH}), 4.01$ (d, $1 \mathrm{H}, J=3.7 \mathrm{~Hz}$, pyrane-H-4), 6.57-7.88 (m, 7H, Ar- $\mathrm{H}), 9.53$ (br, $2 \mathrm{H}, \mathrm{NH}_{2}$ ); ${ }^{13} \mathrm{C}$ NMR: 50.1, 63.8, 113.7, 117.1, 118.4, $121.1,124.2,124.4,129.1,130.3,136.5,146.1,150.8$, 151.2, 176.3; Anal. Calc. $\mathrm{C}_{19} \mathrm{H}_{11} \mathrm{ClN}_{6} \mathrm{O}$ (374.79): C, 60.89; $\mathrm{H}$, 2.96; N, 22.42; Found: C, 60.46; H, 2.54; N, 22.42.

\section{SYNTHESIS OF ETHYL 2-(2-AMINO-6-((4-CHLORO- PHENYL)DIAZENYL)-3-CYANO-4H-CHROMEN-4-YL)-2- CYANOACETATE (7)}

A solution of compound $5(0.01 \mathrm{~mol})$ and ethyl cyanoacetate $(0.01 \mathrm{~mol})$ in a mixture of $15 \mathrm{~mL} \mathrm{EtOH}$ and $5 \mathrm{~mL}$ DMF and $0.5 \mathrm{~mL}$ of piperidine was heated under reflux for
$4 \mathrm{~h}$. The solid that formed was filtered and recrystallized from ethanol as brown crystals. Yield 50 \%; m.p. 250$251^{\circ} \mathrm{C}$; IR: $v / \mathrm{cm}^{-1}=3364,3260\left(\mathrm{NH}_{2}\right), 2990$ (aliphatic $\mathrm{CH}$ ), $2203(\mathrm{C} \equiv \mathrm{N}), 1737$ ( $\mathrm{C}=\mathrm{O}$ ester); ${ }^{1} \mathrm{H}$ NMR: $\delta / \mathrm{ppm}=1.07$ (t, $\left.3 \mathrm{H}, J=7.5 \mathrm{~Hz}, \mathrm{CH}_{3}\right), 3.93(\mathrm{~d}, 1 \mathrm{H}, J=3.8 \mathrm{~Hz}, \mathrm{CH}), 4.16(\mathrm{~d}$, $1 \mathrm{H}, J=3.8 \mathrm{~Hz}, \mathrm{CH}$-pyrane), 4.21 (q, $2 \mathrm{H}, J=7.5 \mathrm{~Hz}, \mathrm{CH}_{2}$ ester), 7.6-8.22 (m, 7H, Ar-H), $9.22\left(\mathrm{br}, 2 \mathrm{H}, \mathrm{NH}_{2}\right) ;{ }^{13} \mathrm{C}$ NMR: $14.8\left(\mathrm{CH}_{3}\right), 39.4(\mathrm{CH}), 71.9(\mathrm{CH}), 60.5\left(\mathrm{CH}_{2}\right), 118.4$ $(\mathrm{C} \equiv N), 118.8(\mathrm{C} \equiv \mathrm{N}), 123.9,124.8,127.4,130.2,137.0$, 144.7, 148.9, 166.9 (C=O); Anal. Calc. $\mathrm{C}_{21} \mathrm{H}_{16} \mathrm{ClN}_{5} \mathrm{O}_{3}$ (421.84): C, 59.79; H, 3.82; N, 16.60; Found: C, 59.45; H, 3.64; N, 16.02 .

\section{SYNTHESIS OF ETHYL 6-((4-CHLOROPHENYL)DIAZENYL)-2- OXO-2H-CHROMENE-3-CARBOXYLATE (8)}

A mixture of compound 1 (0.01 mol), diethyl malonate $(0.01 \mathrm{~mol})$ and catalytic amount of piperidine in EtOH/DMF $(15 / 5 \mathrm{~mL})$ was heated under reflux for $3 \mathrm{~h}$. The solid product was collected by filtration and recrystallized from ethanol as brown crystals. Yield $75 \%$; m.p. $265-267^{\circ} \mathrm{C}$; IR: $\mathrm{v} / \mathrm{cm}^{-1}=2923$ (CH-aliphatic), $1722(\mathrm{C}=\mathrm{O}) ;{ }^{1} \mathrm{H} \mathrm{NMR}: \delta / \mathrm{ppm}$ $=1.20\left(\mathrm{t}, 3 \mathrm{H} ; J=7.5 \mathrm{~Hz}, \mathrm{CH}_{3}\right), 4.29\left(\mathrm{q}, 2 \mathrm{H}, J=7.5 \mathrm{~Hz}, \mathrm{CH}_{2}\right)$, 7.59-8.20 (m, 8H, 7Ar-H \& H-pyrane); ${ }^{13} \mathrm{C}$ NMR: $15.4,62.4$, $113.5,118.3,119.5,117.8,122.5,123.0,124.4,129.1$, 136.5, 149.4, 150.8, 155.2, 156.2, 167.1; Anal. Calc. $\mathrm{C}_{18} \mathrm{H}_{13} \mathrm{ClN}_{2} \mathrm{O}_{4}: \mathrm{C}, 60.60 ; \mathrm{H}, 3.67 ; \mathrm{N}, 7.85$. Found: $\mathrm{C}, 60.84 ; \mathrm{H}$, $3.53 ; \mathrm{N}, 7.54$.

\section{SYNTHESIS OF 2-IMINO-2H-CHROMENE-3- CARBOXAMIDES 9a,b}

To a solution of 1 (0.01 mol), 2-cyano- $N$-(cyclohexyl)acetamide or 2-cyano- $\mathrm{N}$-(furan-3-ylmethyl)acetamide (0.01 mol) in EtOH/DMF (15/5 mL) and catalytic amount of piperidine was heated under reflux for $4 \mathrm{~h}$, then cooled and poured into ice water. The resulting solid was filtered off, washed with water, dried and crystalized from ethanol.

\section{6-((4-Chlorophenyl)diazenyl)- $\mathrm{N}$-cyclohexyl-2-imino-2H-} chromene-3-carboxamide (9a): Pale yellow crystals. Yield 65 \%; m.p.130-132 ${ }^{\circ} \mathrm{C}$; IR: v/cm ${ }^{-1}=3338(\mathrm{NH}), 2922,2852$ (CH-aliph), 1672 (C=O); ${ }^{1} \mathrm{H}$ NMR: $\delta / p p m=0.8-1.06(\mathrm{~m}, 10 \mathrm{H}$, $\mathrm{CH}_{2}$ cyclohexyl), 2.65-2.71 (m, 1H, CH-N cyclohexyl), 7.57$8.13(\mathrm{~m}, 8 \mathrm{H}, \mathrm{Ar}-\mathrm{H}$ \& H-pyrane) , 8.96, 10.17 (2br, 2H, 2NH; cancelled with $\left.\mathrm{D}_{2} \mathrm{O}\right) ;{ }^{13} \mathrm{C}$ NMR: 23.2, 24.7, 33.1, 49.4, 113.1, $118.3,120.5,120.9,124.1,124.4,128.7,129.1,137.5$, 146.2, 151.2, 157.0, 161.3, 164.4; Anal. Calc. $\mathrm{C}_{22} \mathrm{H}_{21} \mathrm{ClN}_{4} \mathrm{O}_{2}$ (408.89): C, 64.62; H, 5.18; N, 13.70. Found: C, 64.85; H, $5.33 ; \mathrm{N}, 13.55$.

\section{6-((4-Chlorophenyl)diazenyl)- $\mathrm{N}$-(furan-2-ylmethyl)-2-} imino-2H-chromene-3-carboxamide (9b): Yellow crystals. Yield $78 \%$; m.p. $230-232^{\circ} \mathrm{C}$; IR: $v / \mathrm{cm}^{-1}=3310,3202(2 \mathrm{NH})$, 2928 (CH-aliph), 1670 (C=O); ${ }^{1} \mathrm{H}$ NMR: $\delta / p p m=4.30$ (s, $2 \mathrm{H}$, $\mathrm{CH}_{2}$ ), 6.39 (d, $1 \mathrm{H}, \mathrm{CH}$-furfuryl), 6.59 (d, $1 \mathrm{H}, \mathrm{CH}$-furfuryl), 7.14-8.03 (m, H, 7Ar-H \& CH-O furfuryl \& H-pyrane), 8.52, 
10.17 (2br, $2 \mathrm{H}, 2 \mathrm{NH}$ cancelled with $\left.\mathrm{D}_{2} \mathrm{O}\right) ;{ }^{13} \mathrm{C}$ NMR: 37.4, $111.2,111.9,112.4,116.2,120.8,121.4,124.5,125.2$, $128.2,130.2,136.5,142.4,145.8,148.2,151.8,156.8$, 160.2, 164.3; Anal. Calc. $\mathrm{C}_{21} \mathrm{H}_{15} \mathrm{ClN}_{4} \mathrm{O}_{3}$ (406.83): C, 62.00; $\mathrm{H}$, 3.72; N, 13.77. Found: $\mathrm{C}, 62.33 ; \mathrm{H}, 3.85 ; \mathrm{N}, 13.54$.

\section{SYNTHESIS OF 6-((4-CHLOROPHENYL)DIAZENYL)-2- IMINO-N'-(4-METHOXYBENZYLIDENE)-2H-CHROMENE-3- CARBOHYDRAZIDE (10)}

To a solution of compound $\mathbf{1}(0.01 \mathrm{~mol})$ and 2-cyano- $N$ '-(4methoxybenzylidene)acetohydrazide $(0.01 \mathrm{~mol})$ in $20 \mathrm{~mL}$ of EtOH/DMF (3/1), a catalytic amount of piperidine was added. The reaction mixture was heated under reflux for 3 h. The solid product formed on hot, collected by filtration and recrystallized from ethanol/DMF as orange crystals. Yield $82 \%$; m.p. $>300{ }^{\circ} \mathrm{C}$; IR: $v / \mathrm{cm}^{-1}=3301(\mathrm{NH}), 2931(\mathrm{CH}-$ aliph), 1660 (C=O); ${ }^{1} \mathrm{H}$ NMR: $\delta / p p m=3.80\left(\mathrm{~s}, 3 \mathrm{H}, \mathrm{OCH}_{3}\right)$, 6.82-8.60 (m, $12 \mathrm{H}, \mathrm{Ar}-\mathrm{H}$ \& pyrane), $8.75(\mathrm{~s}, 1 \mathrm{H}, \mathrm{N}=\mathrm{CH}), 8.89$, $9.10\left(2 \mathrm{br}, 2 \mathrm{H}, 2 \mathrm{NH}\right.$; cancelled with $\left.\mathrm{D}_{2} \mathrm{O}\right) ;{ }^{13} \mathrm{C} \mathrm{NMR}: 55.8$ $\left(\mathrm{CH}_{3}\right), 114.5,114.8,114.9,115.6,124.2,124.3,124.3$, 124.6, 124.7, 128.9, 129.7, 129.7, 129.8, 129.9, 130.0, 130.1, 130.4, 136.1, 151.0 (C=N), 160.9 (C=O); Anal. Calc. $\mathrm{C}_{24} \mathrm{H}_{18} \mathrm{ClN}_{5} \mathrm{O}_{3}$ (459.89): C, 62.68; $\mathrm{H}, 3.95 ; \mathrm{N}, 15.23$; Found: $\mathrm{C}$, 62.56; H, 3.57; N, 15.44 .

\section{SYNTHESIS OF 6-((4-CHLOROPHENYL)DIAZENYL)-2- IMINO-2H-CHROMENE-3-CARBOXAMIDE (11)}

A solution of compound $1(0.01 \mathrm{~mol})$ and cyanoacetamide (0.01 mol) in $20 \mathrm{~mL}$ EtOH/DMF (3/1) containing $0.5 \mathrm{~mL}$ piperidine was heated under reflux for $4 \mathrm{~h}$. The solid was filtered off and recrystallized from ethanol as pale yellow crystals. Yield $82 \%$; m.p. $270-272{ }^{\circ} \mathrm{C}$; IR: $v / \mathrm{cm}^{-1}=3400$ $\left(\mathrm{NH}_{2}+\mathrm{NH}\right), 1651(\mathrm{C}=0) ;{ }^{1} \mathrm{H}$ NMR: $\delta / \mathrm{ppm}=7.11-8.04(\mathrm{~m}, 8 \mathrm{H}$, Ar-H \& H-pyrane), $8.62\left(\mathrm{br}, 1 \mathrm{H}, \mathrm{NH}\right.$ cancelled with $\left.\mathrm{D}_{2} \mathrm{O}\right)$, 9.04 (br, $2 \mathrm{H}, \mathrm{CONH}_{2}$ cancelled with $\left.\mathrm{D}_{2} \mathrm{O}\right) ;{ }^{13} \mathrm{C} \mathrm{NMR}$ : 113.2, $117.3,120.8,121.0,123.0,124.8,128.1,129.8,137.5$, 146.2, 151.2, 157.1, 165.5, 172.7; Anal. Calc. $\mathrm{C}_{16} \mathrm{H}_{11} \mathrm{ClN}_{4} \mathrm{O}_{2}$ (326.74): C, 58.82; H, 3.39; N, 17.15. Found: C, 58.95; H, $3.65 ; \mathrm{N}, 17.43$.

\section{SYNTHESIS OF 2-AMINO-9-((4-CHLOROPHENYL)- DIAZENYL)-5-IMINO-4-OXO-4,5-DIHYDRO-3H- CHROMENO[3,4-C]PYRIDINE-1-CARBONITRILE (12)}

A mixture of $11(0.01 \mathrm{~mol})$ and malononitrile $(0.01 \mathrm{~mol})$ in $20 \mathrm{~mL}$ EtOH/DMF (3/1) was heated under reflux for $4 \mathrm{~h}$, then cooled and poured into ice water. The resulting solid was filtered off, washed with water, dried and purified by recrystallization from ethanol to give compound $\mathbf{1 2}$ as red crystals. Yield $50 \%$; m.p. $>300{ }^{\circ} \mathrm{C}$; IR: $v / \mathrm{cm}^{-1}=3438,3349$, 3242, $3190\left(\mathrm{NH}_{2} \& 2 \mathrm{NH}\right), 2211(\mathrm{C} \equiv \mathrm{N}), 1642(\mathrm{C}=\mathrm{O}) ;{ }^{1} \mathrm{H} \mathrm{NMR}$ : $\delta / \mathrm{ppm}=4.31\left(\mathrm{br}, 2 \mathrm{H}, \mathrm{NH}_{2}\right), 7.61-8.13(\mathrm{~m}, 7 \mathrm{H}, \mathrm{Ar}-\mathrm{H}), 9.53$ (br, 2H, 2NH cancelled with $\left.\mathrm{D}_{2} \mathrm{O}\right) ;{ }^{13} \mathrm{C}$ NMR: 77.1, 112.5, $113.4,116.8,121.5,121.8,123.6,124.4,129.1,136.5$, 145.7, 151.2, 155, 156.5, 161.1, 162.3, 164.4; Anal. Calc:
$\mathrm{C}_{19} \mathrm{H}_{11} \mathrm{ClN}_{6} \mathrm{O}_{2}$ (390.79): C, 58.40; H, 2.84; N, 21.51; Found: C, 58.65; H, 2.54; N, 21.75.

\section{SYNTHESIS OF 1-ACETYL-9-((4-CHLOROPHENYL)- DIAZENYL)-5-IMINO-2-METHYL-3H-CHROMENO[3,4- C]PYRIDIN-4(5H)-ONE (13)}

A mixture of $11(0.01 \mathrm{~mol})$ and acetyl acetone $(0.01 \mathrm{~mol})$ in $20 \mathrm{~mL}$ EtOH/DMF (3/1) was heated under reflux for $4 \mathrm{~h}$, then cooled and poured into ice water. The resulting solid was filtered off, washed with water, dried and purified by recrystallization from ethanol to give $\mathbf{1 3}$ as pall brown crystals. Yield $66 \%$; m.p. $140-142{ }^{\circ} \mathrm{C}$; IR: $v / \mathrm{cm}^{-1}=3338$ (NH), 2929 (CH-aliph), 1742, 1664 (2C=O); ${ }^{1} \mathrm{H}$ NMR: $\delta / p p m$ $=2.27\left(\mathrm{~s}, 3 \mathrm{H}, \mathrm{CH}_{3}\right), 2.47\left(\mathrm{~s}, 3 \mathrm{H}, \mathrm{CH}_{3}\right), 7.20-8.13(\mathrm{~m}, 7 \mathrm{H}, \mathrm{Ar}-$ $\mathrm{H}), 8.7\left(\mathrm{br}, 2 \mathrm{H}, 2 \mathrm{NH}\right.$ cancelled with $\left.\mathrm{D}_{2} \mathrm{O}\right) ;{ }^{13} \mathrm{C}$ NMR: 21.7 , $32.6,112.7,113.7,118.7,121.5,121.9,123.6,124.4,129.1$, 136.5, 145.2, 149.1, 151.5, 155.4, 157.1, 162.5, 164.5, 175.7; Anal. Calc: $\mathrm{C}_{21} \mathrm{H}_{15} \mathrm{ClN}_{4} \mathrm{O}_{3}$ (406.83): $\mathrm{C}, 62.0 ; \mathrm{H}, 3.72 ; \mathrm{N}$, 13.77; Found: C, $62.83 ; \mathrm{H}, 3.43 ; \mathrm{N}, 13.54$

\section{SYNTHESIS OF 2-AMINO-9-((4-CHLOROPHENYL)- DIAZENYL)-3-(FURAN-2-YLMETHYL)-5-IMINO-4-OXO-4,5- DIHYDRO-3H-CHROMENO[3,4-C]PYRIDINE-1- CARBONITRILE (14)}

A solution of compound $9 \mathrm{~b}(0.01 \mathrm{~mol})$ and malononitrile $(0.01 \mathrm{~mol})$ in EtOH/DMF $(15 / 5 \mathrm{~mL})$ containing catalytic amount of piperidine was heated under reflux for $4 \mathrm{~h}$. The solid was filtered off and recrystallized from ethanol/DMF as yellow crystals. Yield $76 \%$; IR: $v / \mathrm{cm}^{-1}=3437,3343,3228$ $\left(\mathrm{NH}_{2} \& \mathrm{NH}\right), 2931(\mathrm{CH}$-aliph), 2207(C $\equiv \mathrm{N}), 1656(\mathrm{C}=\mathrm{O}) ;{ }^{1} \mathrm{H}$ NMR: $\delta / p p m=4.15\left(\mathrm{~s}, 2 \mathrm{H}, \mathrm{CH}_{2}\right), 5.54\left(\mathrm{br}, 2 \mathrm{H}, \mathrm{NH}_{2}\right), 7.00-8.25$ (m, 10H, Ar-H), $9.53\left(\mathrm{br}, 1 \mathrm{H}\right.$, NH cancelled with $\left.\mathrm{D}_{2} \mathrm{O}\right) ;{ }^{13} \mathrm{C} \mathrm{NMR}$ : 43.1, 77.3, 110.4, 111.7, 112.4, 116.8, 118.8, 120.5, 121.2, 123.9, 124.1, 129.5, 136.4, 142.0, 142.9, 145.1, 148.2, 153.8, 155.4, 156.5, 156.9, 164.7; Anal. Calc. $\mathrm{C}_{24} \mathrm{H}_{15} \mathrm{ClN}_{6} \mathrm{O}_{3}$ (470.87): C, 61.22; H, 3.21; N, 17.85. Found: C, 61.35; H, 3.53; N, 17.43

\section{SYNTHESIS OF 9-((4-CHLOROPHENYL)DIAZENYL)-3- (FURAN-2-YLMETHYL)-2-HYDROXY-5-IMINO-4-OXO-4,5- DIHYDRO-3H-CHROMENO[3,4-C]PYRIDINE-1- CARBONITRILE (15)}

A solution of compound $9 \mathrm{~b}(0.01 \mathrm{~mol})$ and ethyl cyanoacetate $(0.01 \mathrm{~mol})$ in $20 \mathrm{~mL}$ (EtOH/DMF; 15/5 mL) containing catalytic amount of piperidine was heated under reflux for $5 \mathrm{~h}$. The solid was filtered off and recrystallized from ethanol as brown crystals. Yield $72 \%$; m.p. 250-252 ${ }^{\circ} \mathrm{C}$; IR: $v / \mathrm{cm}^{-1}=3364$ (broad, OH), $3228(\mathrm{NH}), 2945(\mathrm{CH}-$ aliph), $2201(\mathrm{C} \equiv \mathrm{N}), 1646(\mathrm{C}=0) ;{ }^{1} \mathrm{H}$ NMR: $\delta / \mathrm{ppm}=4.21(\mathrm{~s}$, $\left.2 \mathrm{H}, \mathrm{CH}_{2}\right), 7.51-8.60(\mathrm{~m}, 10 \mathrm{H}, \mathrm{Ar}-\mathrm{H}), 9.2,9.6(2 \mathrm{br}, 2 \mathrm{H}, \mathrm{NH}$, OH cancelled with $\left.\mathrm{D}_{2} \mathrm{O}\right) ;{ }^{13} \mathrm{C}$ NMR: $56.7,110.8,111.2,112.8$, $113.4,115.8,120.4,121.8,123.4,124.4,130.2,137.5$, 143.1, 146.2, 148.7, 151.8, 154.5, 156.0, 156.9, 165.4, 172; Anal. Calc. $\mathrm{C}_{24} \mathrm{H}_{14} \mathrm{ClN}_{5} \mathrm{O}_{4}$ (471.86): C, 61.09; $\mathrm{H}, 2.99 ; \mathrm{N}$, 14.84. Found: $\mathrm{C}, 61.31 ; \mathrm{H}, 2.57 ; \mathrm{N}, 14.77$ 


\section{REFERENCES}

[1] S. A. Hessein, M. A. M. El-Sharief, S. Y. Abbas, H. Kh. Thabet, Y. A. Ammar, Croat. Chem. Acta 2016, 89, 91.

[2] B. S. Kuarm, Y. T. Reddy, J. V. Madhav, P. A. Crooks, B. Rajitha, Bioorg. Med. Chem. Lett. 2011, 21, 524

[3] R. Pratap, V. J. Ram, Tetrahedron 2017, 73, 2529.

[4] A. Ustalar, M. Yilmaz, A. Osman, S. A. Kecel, Turk. J. Chem. 2017, 41, 80.

[5] A. Latifa, K. Mohamed, C. Geffroy-Rodier, G. Jérôme, Z. Sami, Pharmacognosy Communications. 2015, 5, 237.

[6] Y. K. Al-Majedy, A. H. Kadhum, A. A. Al-Amiery, A. Mohamad, Sys, Rev Pharm. 2017, 8, 62.

[7] D. Bhavsar, J. Trivedi, S. Parekh, M. Savant, S. Thakrar, A. Bavishi, A. Radadiya, H. Vala, J. Lunagariya, M. Parmar, L. Paresh, R. Loddo, A. Shah, Bioorg. Med. Chem. Lett. 2011, 21, 3443.

[8] N. R. Kamdar, D. D. Haveliwala, P. T. Mistry, S. K. Patel, Med. Chem. Res. 2012, 20, 854.

[9] O. M. Singh, N. S. Devi, D. S. Thokchom, G. J. Sharma, Eur. J. Med. Chem. 2010, 45, 2250.

[10] B. C. Raju, R. N. Rao, P. Suman, P. Yogeeswari, D. Sriram, T. B. Shaik, S. V. Kalivendi, Bioorg. Med. Chem. Lett. 2011, 21, 2855.

[11] T. Raj, R. K. Bhatia, A. Kapur, M. Sharma, A. K. Saxena, M. P. S. Ishar, Eur. J. Med. Chem. 2010, 45, 790.

[12] K. V. Sashidhara, J. N. Rosaiah, G. Bhatia, J. K. Saxena, Eur. J. Med. Chem. 2008, 43, 2592.

[13] Z. Nazarian, S. Emami, S. Heydari, S. K. Ardestani, M. Nakhjiri, F. Poorrajab, A. Shafiee, A. Foroumadi, Eur. J. Med. Chem. 2010, 45, 1424.
[14] P. Gebhardt, K. Dornberger, F. A. Gollmick, U. Grafe, A. Hartl, H. Gorls, B. Schlegela, C. Hertwecka, Bioorg. Med. Chem. Lett. 2007, 17, 2558.

[15] C. Park, J. Lim, Y. Lee, B. Lee, S. Kim, J. Lee, S. Kim, Enzy. Microb. Tech. 2007, 40, 1758.

[16] G. Chandravadivelu, P. Senniappan, Int. J. Res. Pharm. Chem. 2011, 1, 1082.

[17] S. Concilio, L. Sessa, A. M. Petrone, A. Porta, R. Diana, P. lannelli, S. Piotto, Molecules 2017, 22, 875.

[18] A. Thoraya, Z.A Abdallah, Arkivoc 2008, 17, 295.

[19] Y. A. Ammar, M. A. M. Sh. El-Sharief, M. M. Ghorab, Y. A. Mohamed, A. Ragab, S. Y Abbas, Cur. Org. Syn. 2016, 13, 466.

[20] M. F. El Shehry, M. M. Ghorab, S. Y. Abbas, E. A. Fayed, S. A. Shedid, Y. A. Ammar, Eur. J. Med. Chem., 2018, 143, 1463.

[21] M. A. M. Sh. El-Sharief, S. Y. Abbas, M. A. Zahran, Y. A. Mohamed, A. Ragab, Y. A. Ammar, Z. Naturforschung 2016, 71, 875.

[22] Z. Moussa, M. A. M. S. El-Sharief, S. Y. Abbas, Eur. J. Med. Chem. 2016, 122, 419.

[23] B. S. Furniss, A. J. Hannaferd, and V. Rogers, Vogel's Textbook of Practical Organic Chemistry, Longman, Inc., New York, NY, USA, 4th edition, 1981.

[24] A. A. Farag, Der Pharma Chemica 2015, 7(11), 130

[25] S. A. Fouad, Int. J. Adv. Res. 2014, 2, 442.

[26] R. E. Cooper, Analytical Microbiology, Ed. Kavangeh, F.W. Vol. 1\&2, Academic press, New York and London, 1972.

[27] Clinical and Laboratory Standards Institute (CLSI, formerly National Committee for Clinical and Laboratory Standards NCCLS); Method M27-A2, $2^{\text {nd }}$ Edition; Wayne, Ed.; NCCLS: Pennsylvania, 22 (2002) 1-29. 